\title{
Chemoenzymatic synthesis of daptomycin analogs active against daptomycin-resistant strains
}

\author{
Erin M. Scull ${ }^{1}$. Chandrasekhar Bandari ${ }^{1}$ - Bryce P. Johnson ${ }^{1}$ - Eric D. Gardner ${ }^{1}$ - Marco Tonelli ${ }^{2}$ Jianlan You $^{1}$. \\ Robert H. Cichewicz ${ }^{1} \cdot$ Shanteri Singh ${ }^{1}$ (D)
}

Received: 21 May 2020 / Revised: 21 May 2020 / Accepted: 19 July 2020 / Published online: 28 July 2020

(C) The Author(s) 2020

\begin{abstract}
Daptomycin is a last resort antibiotic for the treatment of infections caused by many Gram-positive bacterial strains, including vancomycin-resistant Enterococcus (VRE) and methicillin- and vancomycin-resistant Staphylococcus aureus (MRSA and VRSA). However, the emergence of daptomycin-resistant strains of S. aureus and Enterococcus in recent years has renewed interest in synthesizing daptomycin analogs to overcome resistance mechanisms. Within this context, three aromatic prenyltransferases have been shown to accept daptomycin as a substrate, and the resulting prenylated analog was shown to be more potent against Gram-positive strains than the parent compound. Consequently, utilizing prenyltransferases to derivatize daptomycin offered an attractive alternative to traditional synthetic approaches, especially given the molecule's structural complexity. Herein, we report exploiting the ability of prenyltransferase CdpNPT to synthesize alkyl-diversified daptomycin analogs in combination with a library of synthetic non-native alkyl-pyrophosphates. The results revealed that CdpNPT can transfer a variety of alkyl groups onto daptomycin's tryptophan residue using the corresponding alkyl-pyrophosphates, while subsequent scaled-up reactions suggested that the enzyme can alkylate the $\mathrm{N} 1, \mathrm{C} 2, \mathrm{C} 5$, and $\mathrm{C} 6$ positions of the indole ring. In vitro antibacterial activity assays using 16 daptomycin analogs revealed that some of the analogs displayed 2-80-fold improvements in potency against MRSA, VRE, and daptomycin-resistant strains of S. aureus and Enterococcus faecalis. Thus, along with the new potent analogs, these findings have established that the regio-chemistry of alkyl substitution on the tryptophan residue can modulate daptomycin's potency. With additional protein engineering to improve the regio-selectivity, the described method has the potential to become a powerful tool for diversifying complex indole-containing molecules.
\end{abstract}

\section{Key points}

- CdpNPT displays impressive donor promiscuity with daptomycin as the acceptor.

- CdpNPT catalyzes N1-, C2-, C5-, and C6-alkylation on daptomycin's tryptophan residue.

- Differential alkylation of daptomycin's tryptophan residue modulates its activity.

Keywords Biocatalysis $\cdot$ Enzyme promiscuity $\cdot$ Tryptophan $\cdot$ Indole $\cdot$ Daptomycin

Erin M. Scull and Chandrasekhar Bandari contributed equally to this work.

Electronic supplementary material The online version of this article (https://doi.org/10.1007/s00253-020-10790-x) contains supplementary material, which is available to authorized users.

Shanteri Singh

shanteri.singh@ou.edu

1 Department of Chemistry and Biochemistry, Stephenson Life Sciences Research Center, University of Oklahoma, 101 Stephenson Parkway, Norman, OK 73019, USA

2 National Magnetic Resonance Facility at Madison, University of Wisconsin-Madison, 411 Babcock Drive, Madison, WI 45005, USA

\section{Introduction}

Daptomycin (Dap, Fig. 1a) belongs to a family of calciumdependent lipodepsipeptide antibiotics produced by Streptomyces roseosporus (Debono et al. 1987; Miao et al. 2005). It is active against several resistant strains of Grampositive bacteria, including methicillin-resistant Staphylococcus aureus (MRSA), vancomycin-intermediate Staphylococcus aureus (VISA), vancomycin-resistant Staphylococcus aureus (VRSA), and vancomycin-resistant Enterococci (VRE) (Steenbergen et al. 2005; Tally and Debruin 2000). As such, Dap (Cubicin $\AA$ ) was approved in 2003 for the treatment of 


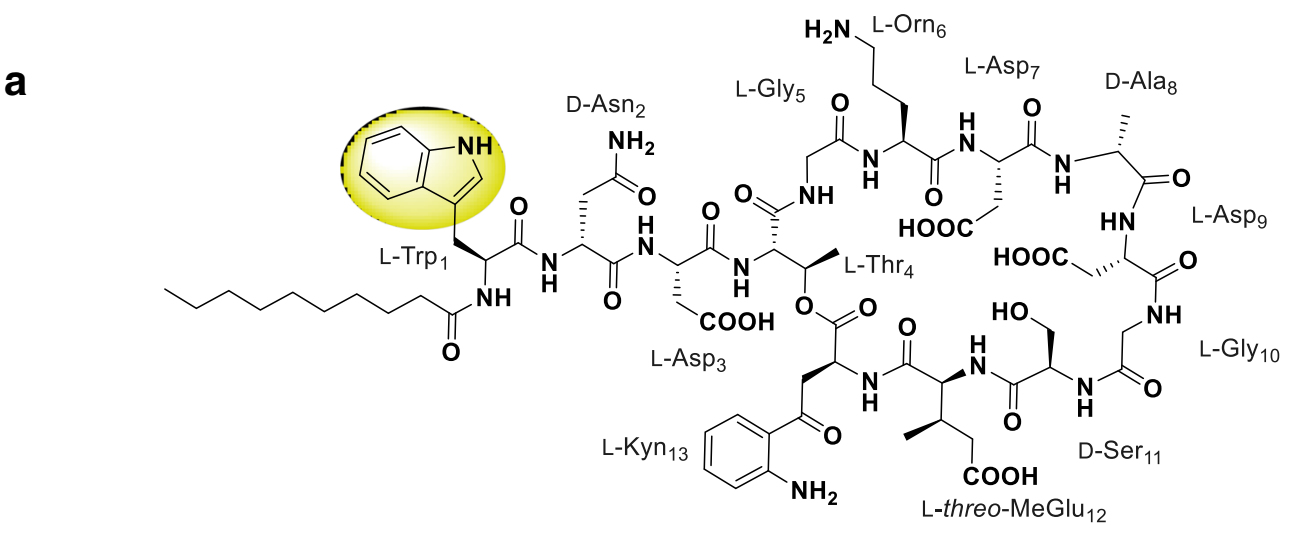

\section{Daptomycin (Dap)}

\section{b} Previously reported Trp modified Dap analogs

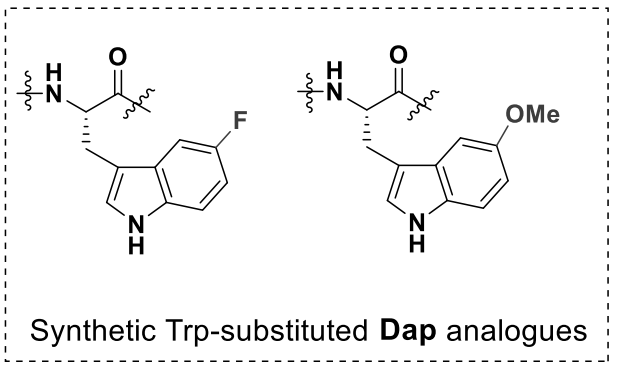

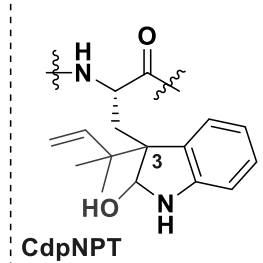

PT-catalyzed synthesis of prenyl-Dap analogues

Fig. 1 a Structure of Dap highlighting Trp residue of interest to the current study in yellow. b Structures of previously reported Trp-modified analogs of Dap (Elshahawi et al. 2017; He et al. 2012)

complicated skin and soft tissue infections, and subsequently in 2006 for S. aureus bacteremia and right-sided infective endocarditis (Arbeit et al. 2004; Chan Tompkins and Harnicar 2008; Eisenstein et al. 2010; Fowler et al. 2006; Sakoulas 2009). Dap is also one of the most promising drugs currently under evaluation for the treatment of pneumococcal meningitis (Cottagnoud et al. 2004; Muri et al. 2018), and while it is active against Streptococcus pneumoniae, it does not meet non-inferiority criteria for the treatment of community-acquired pneumonia due to inhibition by lung surfactants (Silverman et al. 2005). These potent antibacterial activities have been traced back to Dap's complex structure (Fig. 1a) leading to its unique mechanism of action (MOA), which has recently been shown to involve binding to regions of increased fluidity (RIFs) within the Gram-positive cell membrane (Müller et al. 2016). Dap was believed to rigidify the RIFs immediately upon binding and thereby interrupt protein-lipid interactions that rely on the regions' increased fluidity (Gray and Wenzel 2020; Müller et al. 2016; Pogliano et al. 2012). De-anchoring of the lipid II synthase MurG and the phospholipid synthase PlsX was proposed to hinder synthesis of the cell wall and outer membrane, respectively, resulting in Dap's bactericidal effect (Gray and Wenzel 2020; Müller et al. 2016).
However, several key steps in this process remain unresolved, including the involvement of oligomers in membrane permeabilization (Taylor et al. 2017; Zhang et al. 2014). Furthermore, the emergence of Dap-resistant (DapR) S. aureus and Enterococcus (Bayer et al. 2013; Boudjemaa et al. 2018; Goméz Casanova et al. 2017; Pader et al. 2016; Vilhena and Bettencourt 2012) has provided further impetus for developing new derivatives (Barnawi et al. 2018; Doekel et al. 2008; Grünewald et al. 2004; He et al. 2012; Hill et al. 2003; Lam et al. 2013; Lin et al. 2016; Lohani et al. 2015a; Lohani et al. 2015b; Miao et al. 2006; Robbel and Marahiel 2010; Siedlecki et al. 2003) to pursue more extensive structure-activity relationship (SAR) studies and circumvent resistance mechanisms. Several biosynthetic (Doekel et al. 2008; Miao et al. 2006; Robbel and Marahiel 2010), chemoenzymatic (Grünewald et al. 2004), and solid- and solution-phase strategies (Barnawi et al. 2018; He et al. 2012; Hill et al. 2003; Lam et al. 2013; Lin et al. 2016; Lohani et al. 2015a; Lohani et al. 2015b; Siedlecki et al. 2003) have indeed been developed for Dap synthesis and optimization. Despite this progress, only a limited variety of analogs have been prepared, and most of them encompass specific amino acid mutations and modifications of the lipid 
chain or the $\delta$-amino group of ornithine (Barnawi et al. 2018; Doekel et al. 2008; Grünewald et al. 2004; Hill et al. 2003; Lam et al. 2013; Lin et al. 2016; Lohani et al. 2015a; Lohani et al. 2015b; Miao et al. 2006; Robbel and Marahiel 2010; Siedlecki et al. 2003; Yin et al. 2015). While some acylmodified analogs of Dap exhibited improved in vitro activity against DapR strains (Yin et al. 2015), a new strategy with the ability to provide additional structural diversity will enable further optimization of the Dap scaffold and the generation of even more analogs potent against DapR bacteria.

In moving toward such strategies, recent studies have demonstrated replacement of Trp1 on Dap (Fig. 1b) with certain unnatural aromatic amino acids, and/or substitution on the indole ring of Trp1, resulted in analogs with activity against S. aureus (Elshahawi et al. 2017; He et al. 2012). These studies thus establish Trp1 as an important target within Dap for analog synthesis. However, a systematic study of Trp1-based modifications has remained elusive due to the lack of efficient synthetic strategies for such complex scaffolds. Therefore, a new method is needed in general to introduce additional diversity on Dap and enable systematic SAR studies, while one capable of selectively and efficiently modifying the Trp on Dap is perhaps even more urgently needed.

Within this context, a class of natural product enzymes known as aromatic prenyltransferases (PTs) demonstrates broad substrate specificities and is known to modify diverse positions of the indole moiety within complex natural products (Elshahawi et al. 2017; Tanner 2015; Tello et al. 2008). Furthermore, three indole PTs (FgaPT2, CdpNPT, and PriB) have been shown to specifically prenylate Dap using dimethylallyl pyrophosphate as the prenyl donor (Elshahawi et al. 2017). Among the three, CdpNPT demonstrated the highest yield of total product synthesis, and the reaction's major product (N1-Trp-prenyl-Dap) demonstrated higher potency against $S$. aureus compared with the parent compound (Elshahawi et al. 2017). Because PTs are also known to display donor substrate flexibilities (Bandari et al. 2019; Bandari et al. 2017; Johnson et al. 2020; Liebhold and Li 2013; Liebhold et al. 2012; Liebhold et al. 2013; Winkelblech et al. 2015; Yu et al. 2015), we hypothesized that Dap diversification could be achieved if CdpNPT utilized a library of non-native alkyl-pyrophosphates (alkyl-PPs) with Dap as an acceptor. If so, the system would provide facile access to a previously unexplored chemical space for Dap, as well as an opportunity to explore the SAR of Trp1-modified Dap analogs.

Therefore, we report herein the expansion of the alkyldonor substrate scope of the indole PT CdpNPT from Aspergillus fumigatus (Schuller et al. 2012) using 38 nonnative alkyl-donors and Dap as an acceptor. We demonstrate CdpNPT to have exceptional flexibility toward alkyl groups and thus present the first reported example of PT-catalyzed alkyl-diversification of Dap using unnatural donors.
Furthermore, 16 alkyl-diversified-Dap analogs were scaledup, purified, and characterized on a semi-preparative scale, confirming alkyl substitution at the $\mathrm{N} 1, \mathrm{C} 2, \mathrm{C} 5$, or C6 positions of Trp (Fig. 2). Subsequent in vitro antibacterial assays of the newly generated Dap analogs revealed some displayed potency against DapR strains of $S$. aureus and Enterococcus faecalis. Significant increases in potency against Dap susceptible (DapS) strains were also observed for several analogs, showing the regiochemistry of alkyl substitution on the Trp residue can modulate Dap's potency. Thus, the study highlighted the plasticity and broad potential of CdpNPT for alkyl-diversification of natural products (NPs) using nonnative substrate analogs. Furthermore, the development of novel Dap analogs has legitimized PT-based diversification of complex NP scaffolds as a viable method of analog synthesis, and the SAR discussed herein could potentially guide the development of optimized antibacterial leads.

\section{Materials and methods}

\section{General materials}

Dap was purchased from TCI (Portland, OR, USA), while all other reagents were purchased from Sigma-Aldrich (St. Louis, MO, USA), Acros (New Jersey, USA), Alfa-Aesar (Ward Hill, MA, USA), or TCI (Portland, OR, USA) and were of reagent grade or better. PD-10 and Ni-NTA superflow columns were purchased from GE Healthcare (Piscataway, NJ). All alkyl-PPs were synthesized as previously reported (Bandari et al. 2019; Bandari et al. 2017; Johnson et al. 2020). The CdpNPT plasmid was generously provided by Prof. Jon S. Thorson (UK, Lexington, KY). All bacterial strains were obtained from the American Type Culture Collection (ATCC) or the Biodefense and Emerging Infections Research Resources Repository (BEI Resources).

\section{General HPLC and MS methods}

Analytical-scale reactions were analyzed, and Dap analogs were purified, on an Agilent 1220 HPLC system equipped with a DAD. Method A: The CdpNPT-catalyzed reactions were monitored on an analytical reverse-phase (RP) HPLC using a Gemini-NX, C-18 (5 $\mu \mathrm{m}, 4.6 \mathrm{~mm} \times 250 \mathrm{~mm})$ column (Phenomenex, Torrance, California, USA) [gradient of $10 \%$ to $45 \% \mathrm{~B}$ for $10 \mathrm{~min}, 45 \%$ to $100 \% \mathrm{~B}$ for $12 \mathrm{~min}, 100 \%$ to $10 \% \mathrm{~B}$ for $1 \mathrm{~min}, 10 \% \mathrm{~B}$ for $7 \min \left(\mathrm{A}=\mathrm{ddH}_{2} \mathrm{O}\right.$ with $0.1 \%$

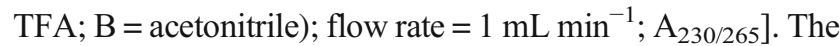
reactions were monitored by the retention time difference between the starting material and the product. Method B: Semipreparative RP HPLC was conducted on a Gemini-NX, C-18 $(5 \mu \mathrm{m}, 10 \mathrm{~mm} \times 250 \mathrm{~mm}$ ) column (Phenomenex, Torrance, California, USA) to purify the Dap analogs [gradient of $10 \%$ 

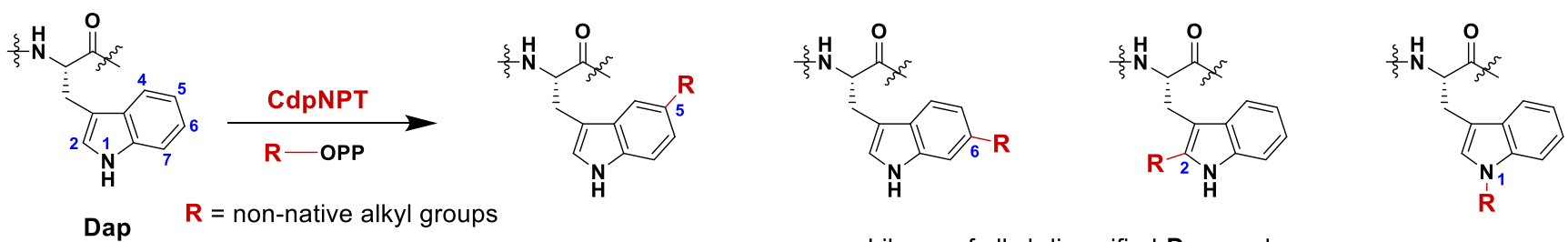

Library of alkyl-diversified-Dap analogs

Fig. 2 This study involving utilization of CdpNPT in the chemoenzymatic generation of Dap analogs with non-native alkyl-PPs (R-OPPs)

to $45 \%$ B for $5 \mathrm{~min}, 45 \%$ to $65 \%$ B for $20 \mathrm{~min}, 65 \%$ to $100 \%$ $\mathrm{B}$ for $5 \mathrm{~min}, 100 \% \mathrm{~B}$ for $2 \mathrm{~min}, 100 \%$ to $10 \% \mathrm{~B}$ for $1 \mathrm{~min}$, $10 \% \mathrm{~B}$ for $7 \min \left(\mathrm{A}=\mathrm{ddH}_{2} \mathrm{O}\right.$ with $0.05 \%$ formic acid; $\mathrm{B}=$

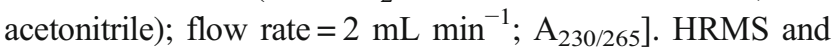
liquid chromatography mass spectrometric (LCMS) data were obtained on an Agilent 6545-QTOF W/1290 HPLC mass spectrometer at the University of Oklahoma Department of Chemistry and Biochemistry.

\section{NMR spectroscopy}

NMR spectra (300-350 $\mu \mathrm{L}$ final volume) were collected at $25^{\circ} \mathrm{C}$ in $5 \mathrm{~mm}$ Shigemi tubes in $99.9 \%$ DMSO- $_{6}$ with $0.05 \%$ $\mathrm{v} / \mathrm{v}$ TMS (Cambridge Isotope Laboratories, Tewksbury, MA, USA) on a 600 or $800 \mathrm{MHz}$ Varian (Palo Alto, CA) VNMRS spectrometer equipped with a $\mathrm{z}$-axis gradient $5 \mathrm{~mm} \mathrm{HCN}$ cold probe at the National Magnetic Resonance Facility at Madison (NMRFAM). For each compound, a set of $1 \mathrm{D}$-proton, ${ }^{1} \mathrm{H}-{ }^{1} \mathrm{H}-$ 2D-COSY, ${ }^{1} \mathrm{H}-{ }^{1} \mathrm{H}-2 \mathrm{D}-\mathrm{TOCSY}$ (mixing time $=80 \mathrm{~ms}$ ), sensitivity-enhanced ${ }^{1} \mathrm{H}_{-}{ }^{13} \mathrm{C}-2 \mathrm{D}-\mathrm{HSQC}(2048 \times 256$ complex data points), ${ }^{1} \mathrm{H}^{-13} \mathrm{C}-2 \mathrm{D}-\mathrm{HMBC}(1024 \times 256$ complex data points), and/or HSQC-TOCSY $(1024 \times 256$ complex data points; with a spin lock field of $7.5 \mathrm{kHz}$ and $24 \mathrm{~ms}$ ) were recorded for resonance assignments. Processing of the spectra was accomplished using the NMRPipe software package (Delaglio et al. 1995). Analysis of NMR data was performed using the NMRFAM-SPARKY software package (Lee et al. 2015).

\section{CdpNPT assays}

CdpNPT was over-expressed and purified as described previously using Ni-NTA chromatography (Elshahawi et al. 2017). Analytical-scale, in vitro CdpNPT reactions were conducted in a volume of $20 \mu \mathrm{L}$ with $1.2 \mathrm{mM}$ alkyl-PP, $1 \mathrm{mM}$ Dap, and $5 \mu \mathrm{M}$ purified CdpNPT in a reaction buffer $(25 \mathrm{mM}$ Tris pH 8.0, $\left.5 \mathrm{mM} \mathrm{CaCl}_{2}, 50 \mathrm{mM} \mathrm{KCl}\right)$. Reactions were incubated at $35{ }^{\circ} \mathrm{C}$ for $16 \mathrm{~h}$ and were quenched by adding an equal volume of methanol. Quenching was followed by centrifugation $(10,000 \times g$ for $15 \mathrm{~min})$ to remove the precipitated protein, and product formation for each reaction was subsequently analyzed by RP-HPLC using method A. For each reaction, percent yield was based upon the integration of species peaks (at $230 \mathrm{~nm}$ or $265 \mathrm{~nm}$ ) and calculated by dividing the integrated area of individual products by the total integrated area of products and remaining substrate. All putative products were subsequently confirmed by HRMS using positive (+) and/or negative (-) modes (see Supporting Information Tables S1 and S2). The Dap reactions with $>30 \%$ product yield were scaled up using standard conditions $(7.5 \mathrm{mM}$ alkyl-PP analog, $5 \mathrm{mM}$ Dap, $5 \mu \mathrm{M}$ CdpNPT, $5 \mathrm{mM} \mathrm{CaCl}_{2}, 50 \mathrm{mM} \mathrm{KCl}$, $25 \mathrm{mM}$ Tris-HCl buffer $\mathrm{pH} 7.5$, total volume $5.0 \mathrm{~mL}$, incubated $16 \mathrm{~h}$ at $35^{\circ} \mathrm{C}$ ) and purified by semi-preparative HPLC using method B. The putative products were confirmed by HRMS (Supporting Information Table S2) and NMR (Supporting Information Table S3).

\section{Antibacterial activity assays}

All Dap analog stocks were calibrated at absorbance $(\mathcal{E}$ $366 \mathrm{~nm}=4000 \mathrm{~L} \mathrm{~mol}^{-1} \mathrm{~cm}^{-1}$ ) (Debono et al. 1988), and all bioactivity assays were conducted in triplicate. All bacterial strains were obtained from the American Type Culture Collection (ATCC) or the Biodefense and Emerging Infections Research Resources Repository (BEI Resources). The strains for which minimum inhibitory concentrations (MICs) were determined included twelve DapS and three DapR strains. The DapS strains were S. aureus (ATCC 25923); S. aureus, MRSA (ATCC 700787); Staphylococcus epidermidis (ATCC 12228); Enterococcus faecalis, VRE (ATCC 700802); Bacillus subtilis (ATCC 6051); S. aureus, VISA, strain SR220 (BEI NR-50512); S. aureus, strain SR1129 (BEI NR50506); S. aureus, strain SR2609 (BEI NR-50507); S. aureus, strain SR2852 (BEI NR-50508); S. aureus, strain SR3777 (BEI NR-50509); S. aureus, strain SR4035 (BEI NR-50510); and Enterococcus faecalis, strain S613 (BEI HM-334). The DapR strains were S. aureus, strain JE2, transposon mutant NE573 (BEI NR-47116); S. aureus, strain JE2, transposon mutant NE1656 (BEI NR-48198); and Enterococcus faecalis, strain R712 (BEI HM-335). MIC testing against all strains was performed in Mueller-Hinton Broth (MHB) medium supplemented with $50 \mathrm{mg} \mathrm{L}^{-1}$ of calcium (MHBc) using NCCLS guidelines for broth microdilution methods and inoculum of $1 \times 10^{5}$ $5 \times 10^{5} \mathrm{CFU} / \mathrm{mL}$ (CLSI: Performance Standards for 
Antimicrobial Susceptibility Testing. 29th ed.). The serial two-fold dilutions of compounds ranged from 5 to $0.039 \mu \mathrm{M}$ and 1.6 to $0.0125 \mu \mathrm{M}$. Briefly, the overnight cultures were grown at $35{ }^{\circ} \mathrm{C}$ in $\mathrm{MHBc}$, while Dap and Dap analogs were serially diluted in MHBc. The serially diluted media was then inoculated with 10,000 counts of bacterial cells from an overnight culture, after which the culture plates were incubated with shaking at $35^{\circ} \mathrm{C}$ for 20 $24 \mathrm{~h}$. Growth was evaluated using the absorbance at $600 \mathrm{~nm}$. The lowest concentration causing 90\% inhibition microbial growth was defined as the MIC, and the concentration units were converted to $\mu \mathrm{g} \mathrm{mL}{ }^{-1}$ in Table 1 for consistency with literature values. Growth and sterility controls were included in each experiment, and two representative Gram-negative bacteria, Escherichia coli (ATCC 11775) and Pseudomonas aeruginosa (ATCC 10145), were included as additional negative controls. The cancer cell-line cytotoxicity assays were used as a measure of general eukaryotic cell toxicity and employed the human hepatocyte carcinoma cell line HepG2 (ATCC HB-8065). The cytotoxicity of Dap and its analogs were tested against the HepG2 cell line at $20 \mu \mathrm{M}$ by using the MTT assay (You et al. 2010).

\section{Results}

\section{Library of non-native alkyl-PPs}

The assessment of CdpNPT-catalyzed alkylation of Dap was carried out using a library of 38 non-native alkyl-PPs synthesized in-house as described previously (1-4, 6-39, Fig. 3) (Bandari et al. 2019; Bandari et al. 2017; Johnson et al. 2020). The assay also included the previously reported natural analog $\mathbf{5}$ for comparison purposes (Elshahawi et al. 2017). Each alkyl-PP contained a double bond at the $\beta$-position (Liebhold and $\mathrm{Li}$ 2013; Liebhold et al. 2012), but beyond this commonality, the synthetic donors represented a wide chemical space. Most of the resulting library consisted of allylic moieties $(\mathbf{1}-\mathbf{2 7}, \mathbf{3 3 - 3 9})$ containing variations in alkyl-chain length, electronic character, and the presence or positioning of branching methyl groups. A small number of analogs were benzylic in nature (28-32) and featured differences in the strength of electron-donating and electronwithdrawing substituents. Additionally, a subset of alkyl-PPs contained moieties amenable to downstream chemoselective reactions (terminal alkenes: 1, 2, 14-16, 26; azides: 33-36; alkynes: 37-39). Thus, the overall library covers a vast chemical space of alkyl-donors not previously explored for CdpNPT.

Table 1 MIC (in $\mu \mathrm{g} \mathrm{mL}^{-1}$ ) of alkyl-Dap analogs against a panel of twelve DapS and three DapR strains (highlighted with yellow background) obtained using a standard microdilution assay as $\mathrm{MIC}_{90}$ after incubation at $35^{\circ} \mathrm{C}$ for $22 \mathrm{~h}$

\begin{tabular}{|c|c|c|c|c|c|c|c|c|c|c|c|c|c|c|c|c|c|}
\hline Strain & DAP & 40 & 41 & 42 & 43 & 44 & 45 & 46 & 47 & 48 & 49 & 50 & 51 & 52 & 53 & 54 & 55 \\
\hline S. aureus (ATCC 25923) & 0.6 & 0.5 & 0.2 & 0.7 & 1.1 & 0.5 & 16.9 & 8.5 & $>34.3$ & 17.1 & 0.2 & 0.5 & 0.5 & 0.1 & 1.1 & 2.8 & 0.3 \\
\hline S. aureus (MRSA, ATCC 700787) & 0.6 & 0.7 & 2.1 & 1.2 & 2.7 & 1.4 & $>33.7>$ & $>34.0$ & $>34.3$ & 4.3 & 2.2 & 2.8 & 2.8 & 2.1 & 2.7 & 2.4 & 0.3 \\
\hline S. epidermidis (ATCC 12228) & 0.3 & 0.5 & 0.5 & 1.2 & 0.5 & 0.5 & 16.9 & 8.5 & 34.3 & 1.1 & 0.5 & 1.1 & 0.7 & 0.5 & 1.1 & 1.1 & 0.4 \\
\hline E. faecalis (hVRE, ATCC 700802) & 1.5 & 2.1 & 0.1 & 0.2 & 0.5 & 1.1 & 33.7 & 8.5 & 34.3 & 0.5 & 0.5 & 0.5 & 0.3 & 0.3 & 1.1 & 0.5 & 1.4 \\
\hline B. subtilis (ATCC 6051) & 2.0 & 8.4 & 2.1 & 2.1 & 1.1 & 2.7 & 4.2 & 4.2 & 8.6 & 2.1 & 2.2 & 2.8 & 2.2 & 2.1 & 2.1 & 2.8 & 2.2 \\
\hline S. aureus (hVISA, SR220) & 4.0 & 0.5 & 0.5 & 0.5 & 1.1 & 0.5 & 33.7 & 17.0 & $>34.3$ & 1.1 & 0.6 & 1.1 & 1.1 & 0.5 & 2.1 & 1.1 & 0.6 \\
\hline S. aureus (SR1129) & 2.0 & 0.3 & 0.5 & 0.3 & 0.7 & 0.3 & 16.9 & 8.5 & 34.3 & 1.4 & 0.3 & 0.6 & 0.6 & 0.3 & 1.4 & 1.1 & 0.6 \\
\hline S. aureus (SR2609) & 4.0 & 2.1 & 0.3 & 0.3 & 1.1 & 0.3 & 16.9 & 8.5 & 34.3 & 1.1 & 0.6 & 0.6 & 0.6 & 0.3 & 2.1 & 1.1 & 0.6 \\
\hline S. aureus (SR2852) & 2.0 & 2.1 & 0.5 & 2.1 & 0.7 & 0.5 & 16.9 & 8.5 & 34.3 & 2.1 & 0.6 & 1.1 & 1.1 & 0.2 & 1.4 & 0.7 & 1.1 \\
\hline S. aureus (hVISA, SR3777) & 2.0 & 2.1 & 0.5 & 2.1 & 1.1 & 0.5 & 16.9 & 8.5 & 34.3 & 1.1 & 1.1 & 1.1 & 0.6 & 0.2 & 1.4 & 1.1 & 2.2 \\
\hline S. aureus (hVISA, SR4035) & 8.1 & 4.2 & 1.4 & 4.3 & 2.1 & 2.1 & $>33.7$ & 8.5 & 2.1 & 2.7 & 2.2 & 2.2 & 1.4 & 1.1 & 8.6 & 2.8 & 4.2 \\
\hline E. faecalis (S613) & 16.2 & 4.2 & 1.1 & 1.1 & 0.5 & 0.5 & $>8.4>$ & $>34.0$ & $>34.3$ & 2.1 & 0.3 & 1.1 & 1.3 & 0.4 & 17.1 & 1.1 & 1.1 \\
\hline E. faecalis (R712) & $>32.4$ & 33.7 & 8.5 & 17.0 & 2.1 & 4.3 & $>8.4>$ & $>34.0$ & $>34.3$ & 17.1 & 2.2 & 17.3 & 5.2 & 6.2 & $>17.1$ & 8.7 & $>34.3$ \\
\hline S. aureus (SAUSA300_1713 NE573) & 16.2 & 4.2 & 0.5 & 2.1 & 0.5 & 2.1 & $>8.4>$ & $>34.0$ & $>34.3$ & 2.1 & 0.5 & 2.2 & 1.3 & 0.4 & 17.1 & 1.1 & 1.1 \\
\hline S. aureus (SAUSA300_1715 NE1656) & 16.2 & 4.2 & 0.5 & 2.1 & 0.5 & 0.5 & $>8.4>$ & $>34.0$ & $>34.3$ & 2.1 & 0.5 & 2.2 & 2.6 & 0.2 & 17.1 & 1.1 & 1.1 \\
\hline
\end{tabular}




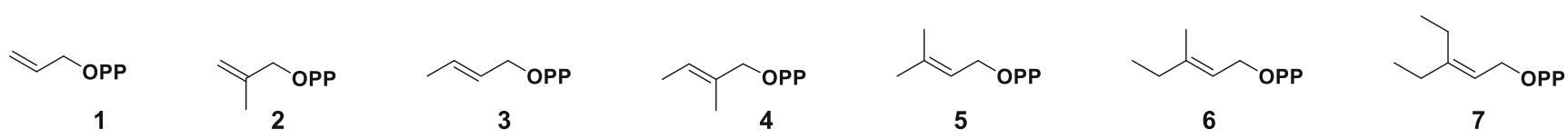

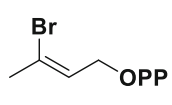

8

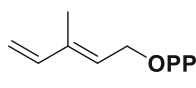

14

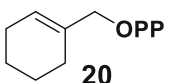

$\overbrace{25}$

$\overbrace{30}^{O P P}$<smiles>C/C(=C\COP)CCN</smiles><smiles>CC(Cl)=CCOP</smiles>

9<smiles>CCCC(=CCOP)CC</smiles>

10

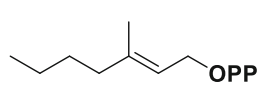

11

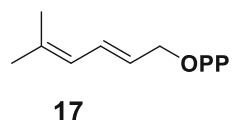

15<smiles>POCC=C(c1ccccc1)c1ccccc1</smiles>

16<smiles>CCCCC/C(C)=C/COP</smiles>

12

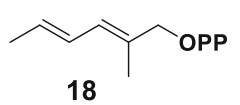

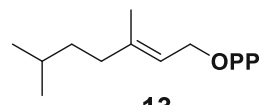

13

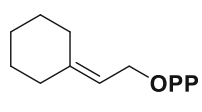

19

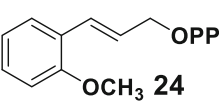

23

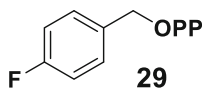

27

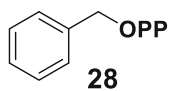<smiles>[NH]C/C=C/CO[Pb]</smiles>

33

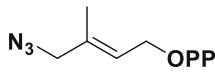

34

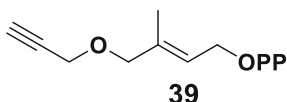

Fig. 3 The alkyl-PP library utilized in this study

\section{Substrate scope of CdpNPT-catalyzed reactions with Dap}

Initial assessment of the CdpNPT-catalyzed alkylation of Dap was illustrated in Fig. 4. Products were detected for 21 of the 39 alkyl-PP analogs $(\mathbf{3}-\mathbf{7}, \mathbf{1 0}, \mathbf{1 4}-\mathbf{2 0}, \mathbf{2 2 - 2 4}, \mathbf{2 6}, \mathbf{2 8}$, 29, 32, 35) and were subsequently confirmed by HRMS analysis (see Supporting Information Table S1). Most of these Dap analogs were mono-alkylated, but in two instances involving 5 and 15, a small amount of dialkylated Dap was also detected and confirmed by HRMS (Supporting Information Table S1). Of the 21 positive reactions, six $(\mathbf{5}, \mathbf{6}, \mathbf{1 5}, \mathbf{1 6}, \mathbf{2 2}$, and $\mathbf{3 2})$ led to appreciable $(>50 \%)$ production of alkyl-Dap derivatives, six (7, 14, 18-20, 24) resulted in moderate $(20-50 \%)$ conversion, and the remaining nine $(\mathbf{3}, \mathbf{4}, \mathbf{1 0}, \mathbf{1 7}, \mathbf{2 3}, \mathbf{2 6}, \mathbf{2 8}, \mathbf{2 9}, \mathbf{3 5})$ offered detectable product formation $(<20 \%)$ under the standard conditions (Fig. 4). Furthermore, the CdpNPTcatalyzed reactions showed a preference for allylic alkylPPs, as 17 (3-7, 10, 14-20, 22-24, 26) of the 21 positive reactions fit this description. The remaining four hits included three benzylic groups $(\mathbf{2 8}, \mathbf{2 9}, \mathbf{3 2})$ and a single reactive handle (the azide $\mathbf{3 5}$ ). Additionally, 10 of the positive allylic reactions $(\mathbf{5}-\mathbf{7}, \mathbf{1 4 - 2 0})$ and the $p$ methoxybenzyl analog (32) resulted in multiple alkylated Dap products, which were indicated by the presence of multiple peaks in the HPLC chromatograms (see Supporting Information Fig. S2). Thus, as with other PTs, CdpNPT shows impressive promiscuity toward non-native donors even in the presence of an unnatural acceptor (Bandari et al. 2019; Bandari et al. 2017; Johnson et al. 2020; Liebhold and Li 2013; Liebhold et al. 2012; Liebhold et al. 2013; Winkelblech et al. 2015; Yu et al. 2015).

\section{Structural characterization of selected alkyl-Dap analogs}

The analytical scale reactions affording $\geq 30 \%$ product yield (Fig. 4, alkyl-PPs 5-7, 15, 16, 18-20, 22, and 32) were scaled up and purified for subsequent characterization using HRMS and NMR spectroscopy. The reaction of 5 produced N1- and C3-regioisomers of Dap in the previous study, with the N1prenylated major product (40) exhibiting increased antibacterial activity compared with Dap (Elshahawi et al. 2017). Thus, we isolated $\mathbf{4 0}$ in the current study and included it as a positive 


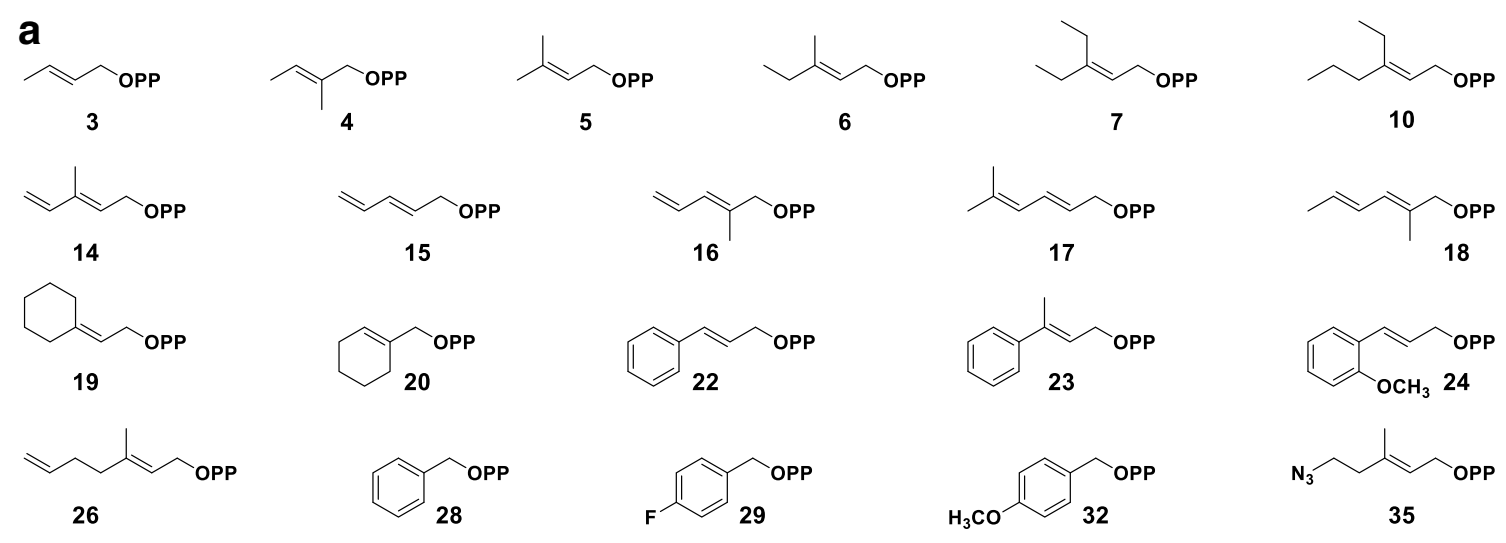

b

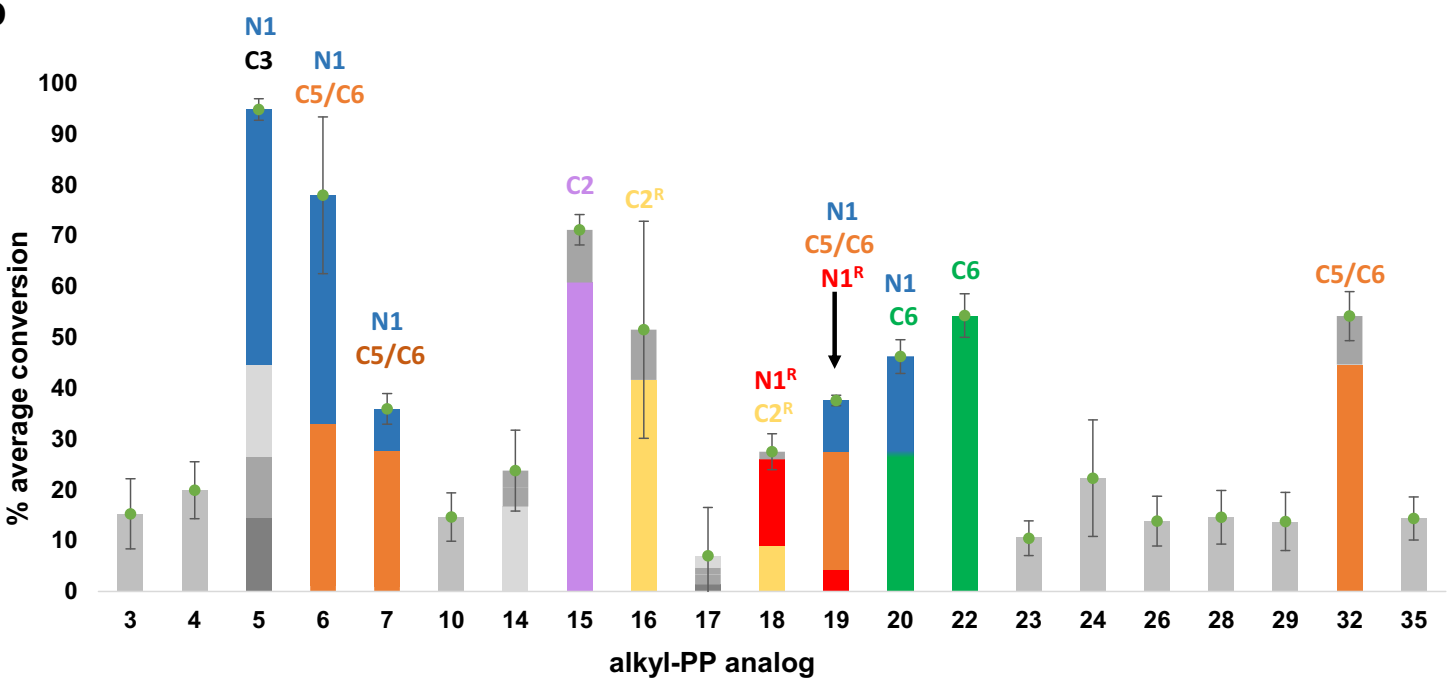

Fig. 4 Donor profile of analytical-scale CdpNPT-catalyzed reactions with Dap. a Structures of analogs whose corresponding reactions gave turnover. b Average percent conversion of all positive reactions with associated standard deviation $(n=3)$ as determined by RP-HPLC (see Supporting Information). Production of individual peaks within each reaction is shown using different colored sections of the full bar. NMRconfirmed structures were given specific colors: blue, $\mathrm{N} 1$; red, $\mathrm{N} 1^{\mathrm{R}}$

control for comparison purposes. As for the non-native alkylPPs, NMR analyses of the alkylated Dap products revealed that CdpNPT catalyzes N1-, C2-, C5- or C6-alkylation of Dap using these donors (Fig. 5). Specifically, N1-alkylation was observed with alkyl-PPs 6, 7, and 18-20; $C 2$-alkylation with 15, 16, and 18; $C 5$-alkylation with 6, 7, 19, and 32; and $C 6$ alkylation with $\mathbf{6}, 7, \mathbf{1 9}, \mathbf{2 0}, \mathbf{2 2}$, and $\mathbf{3 2}$. Thus, $C 2-$ regioisomers were only produced as a major product with diene-containing alkyl-PPs. Furthermore, four of the alkylDap analogs $(\mathbf{4 6}-\mathbf{4 8}, \mathbf{5 0})$ exhibited "reverse" alkylation, a phenomenon observed in the natural CdpNPT reaction in which the new $\mathrm{C}-\mathrm{C}$ or $\mathrm{C}-\mathrm{N}$ bond forms using a carbon atom of the alkyl-PP other than C1' (Fig. 5a) (Bandari et al. 2019; Schuller et al. 2012). The majority of these analogs (46-48) arose from diene-containing alkyl-PPs, though $\mathbf{5 0}$ involved a cyclic derivative of 5 (19). Considered together, these two patterns seem to indicate the existence of active-site (reverse-alkylated); purple, C2; yellow, $\mathrm{C}^{\mathrm{R}}$ (reverse-alkylated); orange, C5/C6 mix; green, C6. Each reaction was carried out in a $20-\mu \mathrm{L}$ volume and contained $1.2 \mathrm{mM}$ alkyl-PP, $1 \mathrm{mM}$ Dap, and $5 \mu \mathrm{M}$ purified CdpNPT in a reaction buffer $\left(25 \mathrm{mM}\right.$ Tris, $\left.\mathrm{pH} 8.0,5 \mathrm{mM} \mathrm{CaCl}_{2}, 50 \mathrm{mM} \mathrm{KCl}\right)$ incubated at $35^{\circ} \mathrm{C}$ for $16 \mathrm{~h}$. No product formation was observed in the absence of CdpNPT or alkyl-PP

interactions prevalent to the conjugated, non-aromatic carbocations of the diene-containing alkyl-PPs. Additionally, while NMR confirmed the purity of most analogs to be $>90 \%$ single regio-isomers, four of the sixteen alkyl-Dap analogs $(41,43,49$, and 55$)$ were found to contain inseparable mixtures of C5-/C6-regioisomers (blue background in Fig. 5b, Supporting Information Table S3). After several attempts to separate these combinations of regioisomers failed, it was decided that any further evaluation of their structures or antibacterial activities would be assessed as mixtures. Notable among these mixtures was the generally lower concentration of $C 5$ regioisomers compared with their $C 6$-counterparts, except in the case of 55. Coupled with the lack of free $C 5$-regioisomers, this pattern could indicate $\mathrm{C} 5$ as a less accessible position on the Trp moiety than either C6 or N1 and more easily reached by benzylic analogs, such as $\mathbf{3 2}$. Thus, while limited by the characterization of only a handful of products, the trends 
a
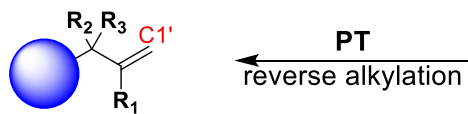

b

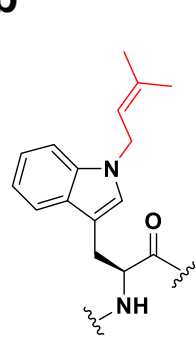

N1-5-Dap

40

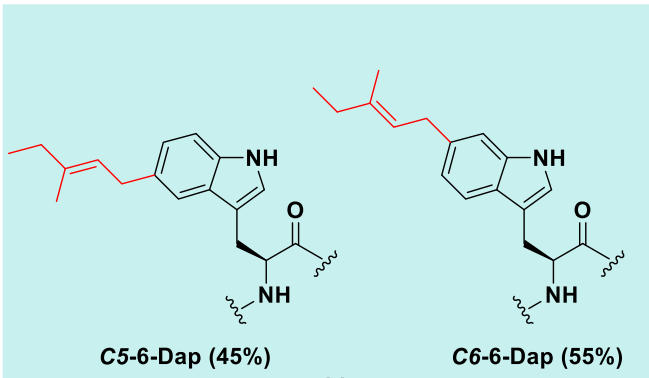

41
$\overbrace{\mathbf{R}_{\mathbf{1}}}^{\mathbf{R}_{\mathbf{2}} \mathrm{OPP}^{\prime}}$

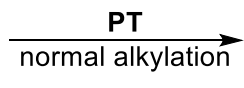

Alkyl-PP
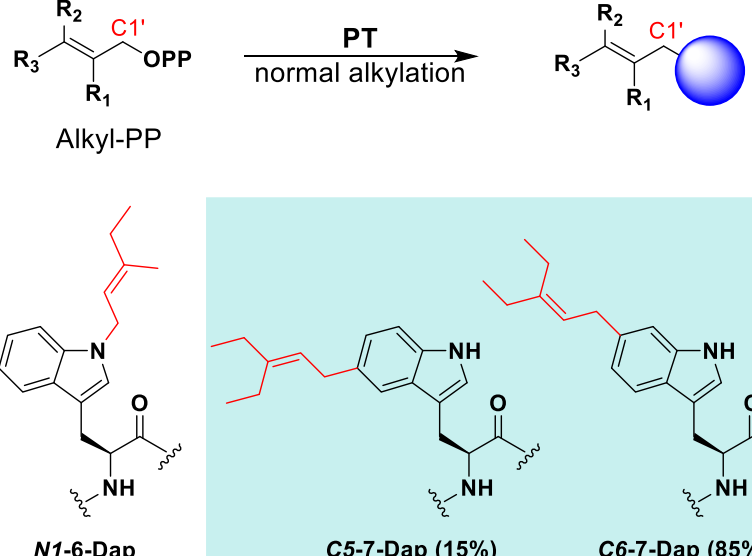

42

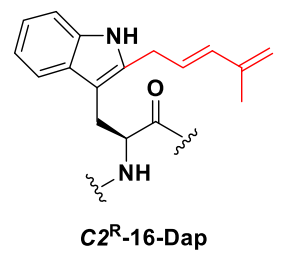

46

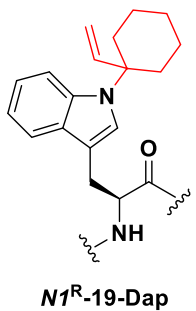

50

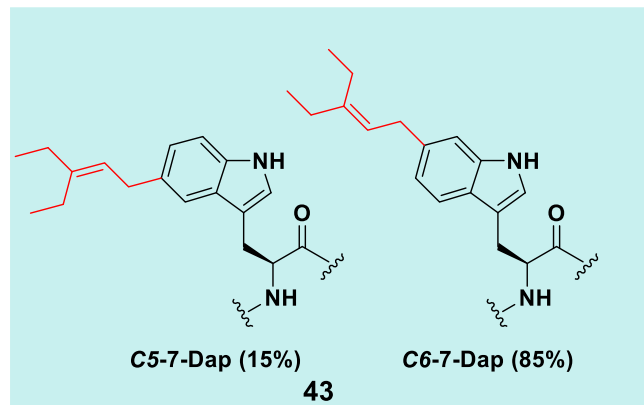

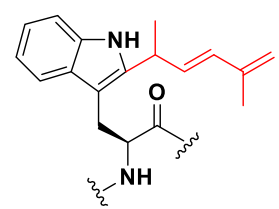

C2 ${ }^{\mathrm{R}}$-18-Dap

47

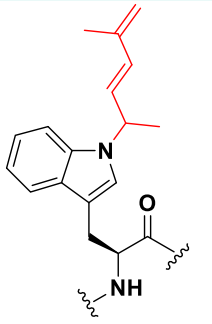

$N 1^{R}$-18-Dap

48<smiles>CCN[C@@H](Cc1cn(CC=C(CC)CC)c2ccccc12)C(=O)O</smiles>

N1-7-Dap

44

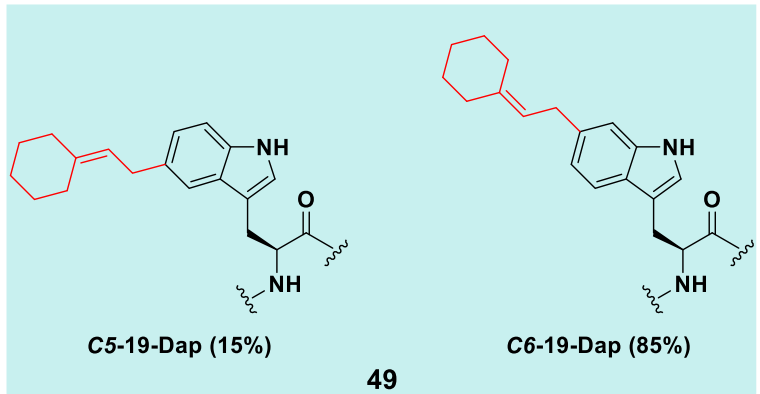

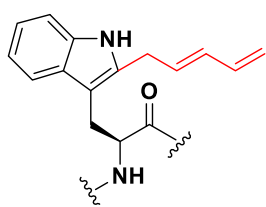

C2-15-Dap 45

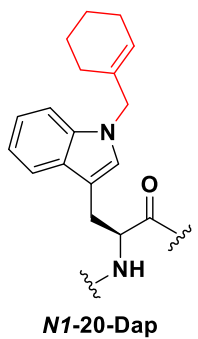

53

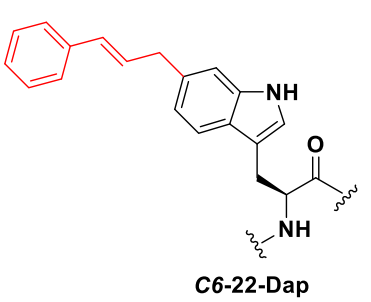

54

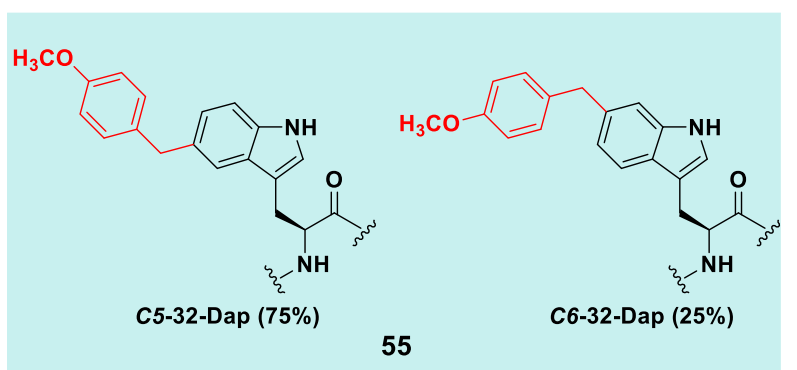

Fig. 5 a Generalized scheme showing PT-catalyzed normal and reverse alkylations of an alkyl-acceptor. b NMR-characterized structures of alkylDap analogs. Blue-boxed structures represent inseparable mixtures of

observed here offer initial considerations for future engineering work in pursuit of regio-specifically modified alkyl-Dap analogs. Additionally, the compounds isolated here provide a novel example of a chemoenzymatic scheme capable of alkylating the relatively inert benzene carbons of the indole moiety on a complex NP.
C5-/C6-regio-isomers (see Supporting Information). Small Rs indicate reverse-alkylated products

\section{Antibacterial activity of purified alkyl-Dap analogs}

Once fully characterized, the entire set of alkyl-Dap analogs was tested for antibacterial activity against a panel of fifteen Gram-positive bacteria, including both DapS and DapR strains, and two Gram-negative controls (Table 1, 
Supporting Information Fig. S1). The assay included the previously reported analog $\mathbf{4 0}$ for comparison purposes (Elshahawi et al. 2017) and the parent Dap as a third control. Additionally, the analogs were tested for general toxicity against eukaryotic cells, which remained largely unchanged compared with Dap, using human hepatocyte carcinoma cell line HepG2. As expected, none of the compounds exhibited antimicrobial activity against Gram-negative $P$. aeruginosa and E. coli. However, several alkyl-Dap analogs possessed significant activity against most of the Gram-positive strains (Table 1, Supporting Information Fig. S1), most notably the C6-, C5-, and N1-substituted analogs. In general, these regioisomers displayed improved activity against DapS S. aureus compared with the parent compound, with $\mathbf{5 2}$ showing the greatest improvement for most strains. In terms of the other DapS strains, significant changes in MIC were not observed for either $S$. epidermidis or B. subtilis, while analogs 41, 42, 51, and 52 displayed a 5-15-fold enhanced activity toward vancomycin-resistant Enterococcus (Table 1, Fig. S1).

Even more interesting, however, were the activities of the C6-, C5-, and N1-substituted analogs with DapR strains. The analogs 41, 43, 49, and 52 showed a MIC range of $0.2-$ $0.5 \mu \mathrm{g} \mathrm{mL}^{-1}$ (Table 1) and a 30-80-fold improvement in antibacterial activity over Dap with DapR S. aureus (Supporting Information Fig. S1). Meanwhile, all except 53 and the $C 2$-alkyl substituted analogs (45-47) had MIC $\leq$ $4 \mu \mathrm{g} \mathrm{mL}^{-1}$ with Enterococcus faecalis strain S613 (Dap MIC of $\left.\sim 16 \mu \mathrm{g} \mathrm{mL}^{-1}\right)$, with the four best analogs $(43,44$, 49, and 52) possessing a MIC range of $0.3-0.6 \mu \mathrm{gL}^{-1}$. Analogs 43 and 49 even displayed MICs of $\sim 2 \mu \mathrm{g} \mathrm{mL}$ against the highly DapR Enterococcus faecalis strain R712 (Table 1). Thus, in general, the most potent alkyl-Dap analogs against DapS and DapR strains were either a single C6-alkylsubstituted Dap (52) or a mixture of $C 5$ - and $C 6$ - regioisomers $(41,43$, and 49). Such large increases in activity among the analogs, especially considering the similar toxicity profile to the parent compound, point to the potential of the alkyl-Dap compounds developed here to become new drug leads. In contrast to these potent Dap analogs, a sharp decrease in overall activity was observed for $C 2$-substituted-Trp analogs (4547) when compared with Dap. A single MIC decrease was observed for one of these analogs among the DapS strains (47 with $S$. aureus SR4035, Table 1), with all other MICs being 2100-fold higher than the parent Dap. Experiments with the DapR strains fared little better, as most of the MIC values for the $C 2$-regioisomers were comparable to or higher than the values for Dap. Taken together, these trends build on previous work (Elshahawi et al. 2017; He et al. 2012) to more firmly establish the effect of Trp-modification on the activity of Dap. Furthermore, several alkyl-Dap analogs produced here have the potential to transition to "drug lead" status based on their potency against both DapS and DapR bacterial strains.

\section{Discussion}

A deeper analysis of the turnover ratios observed in the analytical scale reactions revealed interesting patterns to consider in future studies of CdpNPT. DMAPP (5) provided the highest overall turnover $(94.8 \pm 2.1 \%)$ with Dap, which is unsurprising considering it is the natural donor for CdpNPT. However, the presence of four peaks in the chromatogram implied that the induced fit of donor and acceptor in the active site is not optimal, resulting in low regiospecificity. The structure of CdpNPT has been solved previously (Schuller et al. 2012), so additional crystallographic studies with Dap and $\mathbf{5}$ seem like a plausible next step in the pursuit of optimized enzymes. As for the other analogs, the most successful allylic reactions included analogs very similar to $\mathbf{5}$ in the length of their alkyl-chain (4-6 unbranched carbons: 6, 7, 14 16, 18-20), while several analogs with shorter ( $<4$ carbons: 1 and 2) or longer ( $>6$ carbons: 11-13, 23-27) chains showed 0 $20 \%$ turnover. The binding pocket of CdpNPT has been optimized by nature to utilize DMAPP with indole moieties, so it is highly probable that donors of similar overall structure would also be transferred with relatively similar efficiency.

Unbranched chain length, however, did not seem to be the only steric factor at play. Notably, the conjugated analog series of 21-24 experienced significant losses in activity as larger substituents were added to the core scaffold of 22. For example, the introduction of an $o$-methoxy group (24) substantially lowered the overall turnover. Perhaps even more astonishing was the addition of a methyl group on $\mathrm{C3}^{\prime}$ of $\mathbf{2 3}$, which lowered the turnover even further despite the analog's closeness in structure to $\mathbf{5}$. The bulky phenyl group attached to $\mathrm{C}^{\prime}$ ' of $\mathbf{2 1}$ destroyed activity altogether, further highlighting the impact of steric hindrance in the active site. Additionally, carbocation stabilization likely played a role in substrate acceptance. For example, the removal $(\mathbf{1}, \mathbf{2}, \mathbf{3})$, substitution $(\mathbf{8}$, 9), or movement $(2,4)$ of the branching methyl groups from $\mathrm{C}^{\prime}$ of 5 may explain the lower turnover of the corresponding analogs, as these modifications would likely cause a loss of hyperconjugation. Removal/migration of this methyl group from $\mathrm{C}^{3}$ also removed the possibility of forming tertiary carbocations by resonance, further reducing stability. Thus, while chain length seems to be an acceptable general criterion for predicting allylic donor acceptance, more subtle steric and electronic factors play significant roles as well.

Similar patterns were identified within the other classes of donors. For the benzylated analogs, the substrate scope appeared to arise from an intricate balance between electron donation/withdrawal and steric bulk. The core benzylic scaffold (28) was taken marginally $(14.6 \pm 5.3 \%)$ and the turnover was unaffected by inductive electron withdrawal ( $p$-fluorinated 29, $13.8 \pm 5.7 \%$ ) except for the much bulkier $p$-bromo analog (30). In contrast, the strong electron withdrawing effect of nitro substituents (31) destroyed activity completely, while strong electron donation through delocalization (32) increased 
turnover nearly 4-fold. These patterns indicated a strong dependence of the alkylation reaction on carbocation stability in addition to available space in the active site. As for the analogs with chemoselective functionality, there appeared to be a clear distinction between the types of patterns observed for each subclass. For example, the dienes and the terminal alkene were relatively straightforward. Five of the six dienes were utilized to varying extents, with individual turnovers connected to the presence and placement of methyl groups on the core scaffold of $\mathbf{1 5}$. The bulky phenyl group of $\mathbf{2 5}$, however, appeared to exclude this analog from activity altogether. The terminal alkene $\mathbf{2 6}$ was utilized with relatively low turnover $(13.8 \pm 4.9 \%)$, most likely due to its carbon chain length as described earlier.

The azides, however, presented much more interesting patterns. Only one of the four (35, the longest chain of unbranched carbons) was utilized to a small extent (14.3 \pm 4.3\%). Analog 36 was likely hindered by its relatively higher three-dimensionality compared with the other azides, but the activity pattern of 33-35 was especially surprising considering the more flexible and hydrophobic $\mathbf{1 1}$ and $\mathbf{1 2}$ gave no turnover at all. Using the published ternary complex of CdpNPT (PDB ID: 4E0U) (Schuller et al. 2012), we manually docked DMASPP into the active site to understand the potential interactions that could explain this turnover (Fig. 6). As shown in Fig. 6, there are a considerable number of polar contacts observed within the binding site, several of which are derived from the hydroxyl groups of an ethylene glycol (EG) likely obtained from the crystallization mixture. The contacts included side-chain interactions with Glu116 and Trp419, as well as an interaction with the main-chain carboxyl group of Thr108. Based on the presence of these contacts in the ternary complex, we speculate that the turnover observed for azide $\mathbf{3 5}$ could be derived from a stabilizing interaction with one or more of the polar groups in place of EG. The need for a polar interaction would explain the inability of $\mathbf{1 1}$ and $\mathbf{1 2}$ to turn over, as their hydrophobic tails would be excluded from the EG binding site, while the separation of the EG and DMASPP binding sites could explain why the shorter azides $(\mathbf{3 3}, \mathbf{3 4})$ gave no turnover. Their alkyl-tails may not have been long enough to reach the EG binding site, resulting in their azide groups being unable to form the needed polar contact for stabilization.

As for the alkynes, none of the three (37-39) gave detectable turnover, which likely arose from unfavorable steric interactions in the active site. For example, while $\mathbf{3 7}$ and $\mathbf{3 8}$ were not utilized, their alkene analogs $(\mathbf{1 5}$ and $\mathbf{1 6}$, respectively) were accepted relatively well by CdpNPT. The linear alkyne groups of $\mathbf{3 7}$ and $\mathbf{3 8}$ likely abolished the ability of these analogs to fit easily into the binding site and enter active conformations. The dienes $\mathbf{1 5}$ and $\mathbf{1 6}$, while still having few rotatable bonds, are slightly more flexible than their alkyne counterparts, likely allowing them to enter the active site more easily. As for 39, it most likely suffered from similar inflexibility due to the terminal alkyne, though its inactivity could also be attributed to exclusion from the EG binding site. In terms of chain length, alkyne $\mathbf{3 9}$ has a similar number of unbranched atoms to azide 35, which would imply that it could fit spatially in a similar conformation. However, the presence of the hydrophobic terminal alkyne would disfavor any proximity toward the polar atoms known to interact with EG. Thus, even among analogs with much less variation, activity appeared to stem from the complex interaction of threedimensional structure and electronic stabilization. Such factors will be essential to consider in future alkyl-diversification efforts with both CdpNPT and the PT class as a whole.

Within this same vein, the alteration of a single Dap residue seemed to introduce new structural interactions that significantly affected its activity. The $C 6-, C 5-$, and $N 1$-substituted Dap analogs produced in the current study exhibited modest to impressive increases in antibacterial activity compared with the parent compound. Meanwhile, the $C 2$-substituted

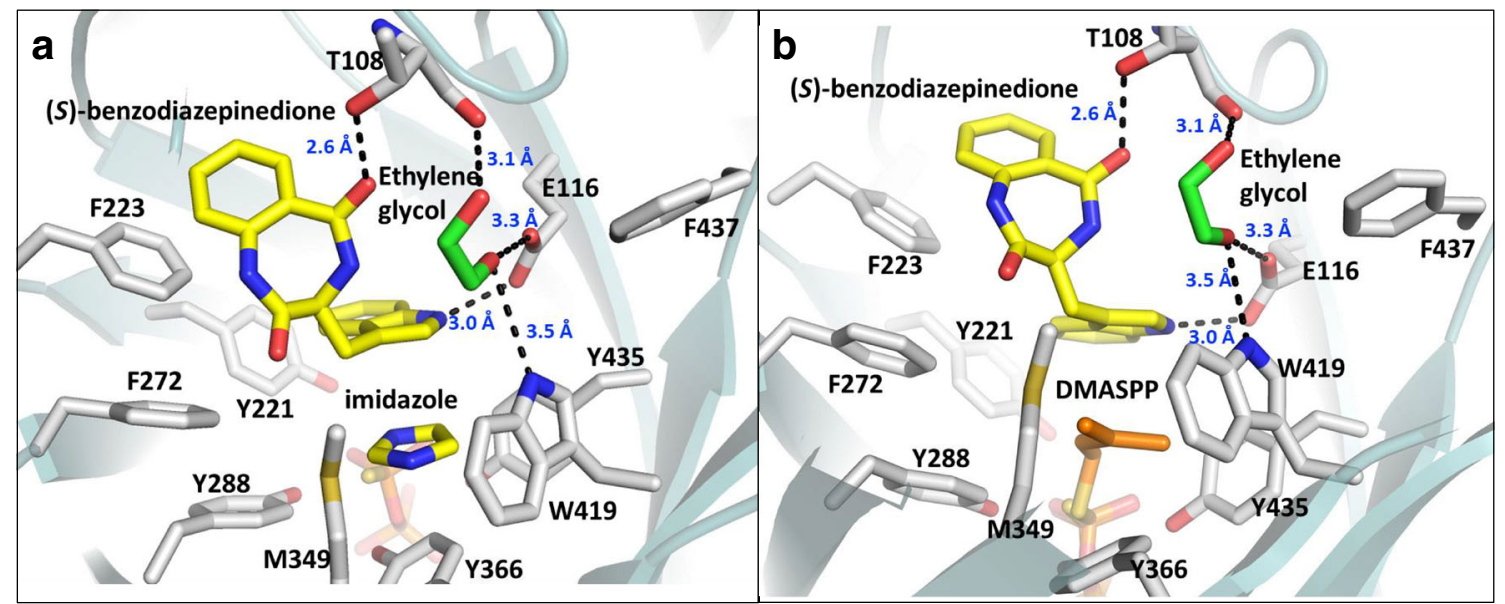

Fig. 6 The crystal structure of CdpNPT (PDB ID: 4E0U) containing either the originally bound imidazole molecule (a) or a manually docked DMASPP molecule (b). Polar interactions are shown as black dashes, and their corresponding lengths (in $\AA$ ) are given in blue 
analogs displayed significantly worse activity than both the other regioisomers as well as unsubstituted Dap. These differences in activity could be due to differences between the structures of Dap and its analogs either in complex with Grampositive membranes or in their unbound form. However, with no three-dimensional structure data for the analogs, only conjecture can be offered as to the cause. Based on the available 3D-NMR data for the parent Dap (Ball et al. 2004; Jung et al. 2004), the benzene ring of Trp is thought to stick out away from the peptide core and lie roughly parallel to the main axis of the acyl tail. Assuming this orientation is maintained upon alkylation of $\mathrm{C} 5$ or $\mathrm{C6}$, the additional hydrophobicity of the added alkyl group could increase the frequency and strength of Dap insertion into the cell membrane, thus increasing the analog's activity. In the case of $N 1$-substitution, the hydrophobic alkyl group may not extend to the same length along the acyl chain as in $C 5$-/C6-substitution, causing a decrease in activity compared with the C5/C6-regioisomers. As for the $C 2$-substituted analogs, new steric strain introduced at the normally inward-facing position could cause a rotation of the indole ring and subsequent change in peptide fold that interferes with oligomerization. A similar argument could be made for the $C 3$-reverse-prenylated Dap compound produced previously (Elshahawi et al. 2017), which also suffered from a decrease in activity compared with its N1-normal-prenylated regioisomer. Conversely, the diene moieties attached at the $\mathrm{C} 2$ position are prone to conjugate addition reactions which would prevent the proper binding conformation or even enable degradation pathways. Therefore, to check their stability, all purified Dap analogs were reinjected on RP-HPLC after their structural and activity characterization (see Supporting Information Fig. S3). Notably, the chromatogram of $\mathbf{4 5}$ seemed to indicate significant degradation of the final product (see Supporting Information Fig. S3). While future kinetic and engineering studies will likely answer these questions, the SAR identified in this study will inform future Dap development efforts using the CdpNPT-based diversification scheme. The C6-, C5-, and N1-substituted Dap analogs developed here even have the potential to progress further in the drug discovery pipeline as lead compounds.

In summary, the current study has provided a proof-ofconcept example of indole functionalization on complex NPs using a PT. Specifically, the late-stage diversification of Dap has been achieved using CdpNPT in conjunction with a library of 39 native and non-native alkyl-PPs, 21 of which resulted in successful production of alkylated product. These analytical-scale reactions outlined the previously unreported donor scope of CdpNPT with Dap as the acceptor, which allowed for preliminary analysis of how steric factors and carbocation stability affected activity. Further scale-up experiments produced 16 alkyl-Dap analogs for structural elucidation by NMR and eventual antibacterial activity assays. The resulting spectra indicated that CdpNPT was capable of alkylating multiple positions on the Trp moiety of Dap (including $\mathrm{N} 1, \mathrm{C} 2, \mathrm{C} 5$, and $\mathrm{C} 6$ ), and the subsequent antibacterial assays produced the first SAR studies of Trp-alkylation of Dap. The results of these experiments revealed the dramatic effect of differential alkylation on an analog's ability to kill Gram-positive strains. In general, N1-, C5-, and C6-substituted analogs showed higher potency against both DapS and DapR strains compared with the parent molecule, while the C2-substituted analogs were much less effective, all possibly due to changes in how the analog interacts with the membrane compared with Dap. Thus, the current study has delivered several Dap analogs effective against DapR bacteria, further strengthening the utility of CdpNPT as a legitimate drug discovery tool. While a lack of regioselectivity currently limits the approach, the advent of protein engineering is likely to improve this aspect of the described methodology. Finally, once the reaction has been optimized with enzyme variants, more in-depth SAR studies and in vivo infection models become much more accessible for the potential drug leads identified here.

Acknowledgments We thank graduate (Dustin A. Dimas and Tejaswi Bavineni) and undergraduate (Johanna M. Masterson, Rachel H. Tran, Andrea L. Batchev, and Abigail F. Lange) students for their participation in the synthesis of alkyl-PP analogs. We gratefully acknowledge the University of Oklahoma Department of Chemistry and Biochemistry NMR and Mass Spectrometry Facilities for analytical support, and ATCC and BEI resources for providing the bacterial strains. This study made use of the National Magnetic Resonance Facility at Madison, which is supported by NIH grant P41GM103399 (NIGMS), old number: P41RR002301. The equipment was purchased with funds from the University of Wisconsin-Madison, the NIH (P41GM103399, S10RR02781, S10RR08438, S10RR023438, S10RR025062, S10RR029220), the NSF (DMB-8415048, OIA-9977486, BIR9214394), and the USDA. Thanks to Jon S. Thorson for graciously providing the prenyltransferase CdpNPT expression strain.

Author contributions SS conceived and designed the research. ES, CB, JY, MT, and SS conducted the experiments. ES, CB, BJ, EG, JY, and SS analyzed the data. RC provided BSL2 facilities and contributed through discussions. BJ and SS wrote the manuscript. All authors read and approved the manuscript.

Funding information This work was supported in part by the University of Oklahoma Faculty Investment grant, an Oklahoma Health Research grant (OCAST, HR19-080), and by the National Institutes of Allergy and Infectious Diseases of the National Institutes of Health under Award Number R03AI141950.

Availability of data and material All data are available upon reasonable request.

\section{Compliance with ethical standards}

Conflict of interest The authors declare that they have no conflicts of interest.

Ethical statements N/A 


\section{Code availability N/A}

Open Access This article is licensed under a Creative Commons Attribution 4.0 International License, which permits use, sharing, adaptation, distribution and reproduction in any medium or format, as long as you give appropriate credit to the original author(s) and the source, provide a link to the Creative Commons licence, and indicate if changes were made. The images or other third party material in this article are included in the article's Creative Commons licence, unless indicated otherwise in a credit line to the material. If material is not included in the article's Creative Commons licence and your intended use is not permitted by statutory regulation or exceeds the permitted use, you will need to obtain permission directly from the copyright holder. To view a copy of this licence, visit http://creativecommons.org/licenses/by/4.0/.

\section{References}

Arbeit RD, Maki D, Tally FP, Campanaro E, Eisenstein BI (2004) The safety and efficacy of daptomycin for the treatment of complicated skin and skin-structure infections. Clin Infect Dis 38(12):16731681. https://doi.org/10.1086/420818

Ball L-J, Goult CM, Donarski JA, Micklefield J, Ramesh V (2004) NMR structure determination and calcium binding effects of lipopeptide antibiotic daptomycin. Org Biomol Chem 2:1872-1878. https://doi. org $/ 10.1039 / b 402722 \mathrm{a}$

Bandari C, Scull EM, Masterson JM, Tran RHQ, Foster SB, Nicholas KM, Singh S (2017) Determination of alkyl-donor promiscuity of tyrosine-O-prenyltransferase $\mathrm{SirD}$ from Leptosphaeria maculans. ChemBioChem 18(23):2323-2327. https://doi.org/10.1002/cbic. 201700469

Bandari C, Scull EM, Bavineni T, Nimmo SL, Gardner ED, Bensen RC, Burgett AW, Singh S (2019) FgaPT2, a biocatalytic tool for alkyldiversification of indole natural products. MedChemComm 10(8): 1465-1475. https://doi.org/10.1039/c9md00177h

Barnawi G, Noden M, Taylor R, Lohani C, Beriashvili D, Palmer M, Taylor SD (2018) An entirely fmoc solid phase approach to the synthesis of daptomycin analogs. Pept Sci:e23094. https://doi.org/ 10.1002/bip.23094

Bayer AS, Schneider T, Sahl HG (2013) Mechanisms of daptomycin resistance in Staphylococcus aureus: role of the cell membrane and cell wall. Ann N Y Acad Sci 1277:139-158. https://doi.org/ 10.1111/j.1749-6632.2012.06819.x

Boudjemaa R, Cabriel C, Dubois-Brissonnet F, Bourg N, Dupuis G, Gruss A, Lévêque-Fort S, Briandet R, Fontaine-Aupart M-P, Steenkeste K (2018) Impact of bacterial membrane fatty acid composition on the failure of daptomycin to kill Staphylococcus aureus. Antimicrob Agents Chemother 62(7):AAC.00023-18. https://doi. org/10.1128/aac.00023-18

Chan Tompkins NH, Harnicar SJ (2008) Prescribing trends with daptomycin (Cubicin) for the treatment of gram-positive infections. Pharm Ther 33(5):282-288

Cottagnoud P, Pfister M, Acosta F, Cottagnoud M, Flatz L, Kuhn F, Muller HP, Stucki A (2004) Daptomycin is highly efficacious against penicillin-resistant and penicillin- and quinolone-resistant Pneumococci in experimental meningitis. Antimicrob Agents Chemother 48(10):3928-3933. https://doi.org/10.1128/aac.48.10. 3928-3933.2004

Debono M, Barnhart M, Carrell CB, Hoffmann JA, Occolowitz JL, Abbott BJ, Fukuda DS, Hamill RL, Biemann K, Herlihy WC (1987) A21978C, a complex of new acidic peptide antibiotics. Isolation, chemistry, and mass spectral structure elucidation. J Antibiot 40(6):761-777. https://doi.org/10.7164/antibiotics.40.761
Debono M, Abbott BJ, Molloy RM, Fukuda DS, Hunt AH, Daupert VM, Counter FT, Ott JL, Carrell CB, Howard LC, Boeck LVD, Hamill RL (1988) Enzymatic and chemical modifications of lipopeptide antibiotic A21978C: the synthesis and evaluation of daptomycin (LY146032). J Antibiot 41(8):1093-1105. https://doi.org/10.7164/ antibiotics.41.1093

Delaglio F, Grzesiek S, Vuister GW, Zhu G, Pfiefer J, Bax A (1995) NMRPipe: a multidimensional spectral processing system based on UNIX pipes. J Biomol NMR 6:277-293. https://doi.org/10. 1007/BF00197809

Doekel S, Coeffet-Le Gal MF, Gu JQ, Chu M, Baltz RH, Brian P (2008) Non-ribosomal peptide synthetase module fusions to produce derivatives of daptomycin in Streptomyces roseosporus. Microbiology 154(Pt 9):2872-2880. https://doi.org/10.1099/mic.0.2008/020685-0

Eisenstein BI, Oleson FB Jr, Baltz RH (2010) Daptomycin: from the mountain to the clinic, with essential help from Francis Tally, MD. Clin Infect Dis 50(Suppl 1):S10-S15. https://doi.org/10.1086/ 647938

Elshahawi SI, Cao H, Shaaban KA, Ponomareva LV, Subramanian T, Farman ML, Spielmann HP, Phillips GN, Thorson JS, Singh S (2017) Structure and specificity of a permissive bacterial Cprenyltransferase. Nat Chem Biol 13(4):366-368. https://doi.org/ 10.1038/nchembio. 2285

Fowler J, VG, Boucher HW, Corey GR, Abrutyn E, Karchmer AW, Rupp ME, Levine DP, Chambers HF, Tally FP, Vigliani GA, Cabell CH, Link AS, De Meyer I, Filler SG, Zervos M, Cook P, Parsonnet J, Bernstein JM, Price CS, Forrest GN, Fätkenheuer G, Gareca M, Rehm SJ, Brodt HR, Tice A, Cosgrove SE (2006) Daptomycin versus standard therapy for bacteremia and endocarditis caused by Staphylococcus aureus. N Engl J Med 355(7):653665. https://doi.org/10.1056/NEJMoa053783

Goméz Casanova N, Siller Ruiz M, Muñoz Bellido JL (2017) Mechanisms of resistance to daptomycin in Staphylococcus aureus. Rev Esp Quimioter 30(6):391-396

Gray DA, Wenzel M (2020) More than a pore: a current perspective on the in vivo mode of action of the lipopeptide antibiotic daptomycin. Antibiotics (Basel) 9(1). https://doi.org/10.3390/antibiotics9010017

Grünewald J, Sieber SA, Mahlert C, Linne U, Marahiel MA (2004) Synthesis and derivatization of daptomycin: a chemoenzymatic route to acidic lipopeptide antibiotics. J Am Chem Soc 126(51): 17025-17031. https://doi.org/10.1021/ja045455t

He Y, Li J, Yin N, Herradura PS, Martel L, Zhang Y, Pearson AL, Kulkarni V, Mascio C, Howland K, Silverman JA, Keith DD, Metcalf CA (2012) Reduced pulmonary surfactant interaction of daptomycin analogs via tryptophan replacement with alternative amino acids. Bioorg Med Chem Lett 22(19):6248-6251. https:// doi.org/10.1016/j.bmcl.2012.08.013

Hill J, Siedlecki J, Parr I, Morytko M, Yu X, Zhang Y, Silverman J, Controneo N, Laganas V, Li T, Lai J-J, Keith D, Shimer G, Finn J (2003) Synthesis and biological activity of $N$-acylated ornithine analogues of daptomycin. Bioorg Med Chem Lett 13(23):4187-4191. https://doi.org/10.1016/j.bmcl.2003.07.019

Johnson BP, Scull EM, Dimas DA, Bavineni T, Bandari C, Batchev AL, Gardner ED, Nimmo SL, Singh S (2020) Acceptor substrate determines donor specificity of an aromatic prenyltransferase: expanding the biocatalytic potential of NphB. Appl Microbiol Biotechnol 104: 4383-4395. https://doi.org/10.1007/s00253-020-10529-8

Jung D, Rozek A, Okon M, Hancock RE (2004) Structural transitions as determinants of the action of the calcium-dependent antibiotic daptomycin. Chem Biol 11(7):949-957. https://doi.org/10.1016/j. chembiol.2004.04.020

Lam HY, Zhang Y, Liu H, Xu J, Wong CTT, Xu C, Li X (2013) Total synthesis of daptomycin by cyclization via a chemoselective serine ligation. J Am Chem Soc 135(16):6272-6279. https://doi.org/10. $1021 / \mathrm{ja} 4012468$ 
Lee W, Tonelli M, Markley JL (2015) NMRFAM-SPARKY: enhanced software for biomolecular NMR spectroscopy. Bioinformatics 31(8):1325-1327. https://doi.org/10.1093/bioinformatics/btu830

Liebhold M, Li S-M (2013) Regiospecific benzylation of tryptophan derivatives catalyzed by a fungal dimethylallyl transferase. Org Lett 15(22):5834-5837. https://doi.org/10.1021/ol4029012

Liebhold M, Xie X, Li S-M (2012) Expansion of enzymatic Friedelcrafts alkylation on indoles: acceptance of unnatural $\beta$-unsaturated allyl diphosphates by dimethylallyl-tryptophan synthases. Org Lett 14(18):4882-4885. https://doi.org/10.1021/ol302207r

Liebhold M, Xie X, Li S-M (2013) Breaking cyclic dipeptide prenyltransferase regioselectivity by unnatural alkyl donors. Org Lett 15(12):3062-3065. https://doi.org/10.1021/ol401247s

Lin DA, Lam HY, Han W, Cotroneo N, Pandya BA, Li X (2016) Structure-activity relationship of daptomycin analogues with substitution at $(2 S, 3 R)-3$-methyl glutamic acid position. Bioorg Med Chem Lett 27:456-459. https://doi.org/10.1016/j.bmcl.2016.12.046

Lohani CR, Taylor R, Palmer M, Taylor SD (2015a) Solid-phase synthesis and in vitro biological activity of a Thr $4 \rightarrow$ Ser4 analog of daptomycin. Bioorg Med Chem Lett 25(23):5490-5494. https://doi.org/ 10.1016/j.bmcl.2015.10.072

Lohani CR, Taylor R, Palmer M, Taylor SD (2015b) Solid-phase total synthesis of daptomycin and analogs. Org Lett 17(3):748-751. https://doi.org/10.1021/acs.orglett.5b00043

Miao V, Coëffet-LeGal M, Brian P, Brost R, Penn J, Whiting A, Martin S, Ford R, Parr I, Bouchard M, Silva CJ, Wrigley SK, Baltz RH (2005) Daptomycin biosynthesis in Streptomyces roseosporus: cloning and analysis of the gene cluster and revision of peptide stereochemistry. Microbiology 151(5):1507-1523. https://doi.org/ 10.1099/mic.0.27757-0

Miao V, Coëffet-Le Gal M-F, Nguyen K, Brian P, Penn J, Whiting A, Steele J, Kau D, Martin S, Ford R, Gibson T, Bouchard M, Wrigley SK, Baltz RH (2006) Genetic engineering in Streptomyces roseosporus to produce hybrid lipopeptide antibiotics. Chem Biol 13(3):269-276. https://doi.org/10.1016/j.chembiol.2005.12.012

Müller A, Wenzel M, Strahl H, Grein F, Saaki TNV, Kohl B, Siersma T, Bandow JE, Sahl HG, Schneider T, Hamoen LW (2016) Daptomycin inhibits cell envelope synthesis by interfering with fluid membrane microdomains. Proc Natl Acad Sci U S A 113(45): E7077-E7086. https://doi.org/10.1073/pnas.1611173113

Muri L, Grandgirard D, Buri M, Perny M, Leib SL (2018) Combined effect of non-bacteriolytic antibiotic and inhibition of matrix metalloproteinases prevents brain injury and preserves learning, memory and hearing function in experimental paediatric pneumococcal meningitis. J Neuroinflammation 15(1):233. https://doi.org/10.1186/ s12974-018-1272-8

Pader V, Hakim S, Painter KL, Wigneshweraraj S, Clarke TB, Edwards AM (2016) Staphylococcus aureus inactivates daptomycin by releasing membrane phospholipids. Nat Microbiol 2:16194. https:// doi.org/10.1038/nmicrobiol.2016.194

Pogliano J, Pogliano N, Silverman JA (2012) Daptomycin-mediated reorganization of membrane architecture causes mislocalization of essential cell division proteins. J Bacteriol 194(17):4494-4504. https://doi.org/10.1128/JB.00011-12

Robbel L, Marahiel MA (2010) Daptomycin, a bacterial lipopeptide synthesized by a nonribosomal machinery. J Biol Chem 285(36): 27501-27508. https://doi.org/10.1074/jbc.r110.128181

Sakoulas G (2009) Clinical outcomes with daptomycin: a post-marketing, real-world evaluation. Clin Microbiol Infect 15(Suppl. 6):11-16. https://doi.org/10.1111/j.1469-0691.2009.03054.x

Schuller JM, Zocher G, Liebhold M, Xie X, Stahl M, Li SM, Stehle T (2012) Structure and catalytic mechanism of a cyclic dipeptide prenyltransferase with broad substrate promiscuity. J Mol Biol 422(1):87-99. https://doi.org/10.1016/j.jmb.2012.05.033

Siedlecki J, Hill J, Parr I, Yu X, Morytko M, Zhang Y, Silverman J, Controneo N, Laganas V, Li T, Li J, Keith D, Shimer G, Finn J (2003) Array synthesis of novel lipodepsipeptide. Bioorg Med Chem Lett 13(23):4245-4249. https://doi.org/10.1016/j.bmcl.2003. 07.025

Silverman JA, I. ML, Van Praagh ADG, Li T, Alder J (2005) Inhibition of daptomycin by pulmonary surfactant: in vitro modeling and clinical impact. J Infect Dis 191(12):2149-2152. https://doi.org/10.1086/ 430352

Steenbergen JN, Alder J, Thorne GM, Tally FP (2005) Daptomycin: a lipopeptide antibiotic for the treatment of serious Gram-positive infections. J Antimicrob Chemother 55(3):283-288. https://doi. org/10.1093/jac/dkh546

Tally FP, Debruin MF (2000) Development of daptomycin for Grampositive infections. J Antimicrob Chemother 46(4):523-526. https://doi.org/10.1093/jac/46.4.523

Tanner ME (2015) Mechanistic studies on the indole prenyltransferases. Nat Prod Rep 32(1):88-101. https://doi.org/10.1039/c4np00099d

Taylor R, Beriashvili D, Taylor S, Palmer M (2017) Daptomycin pore formation is restricted by lipid acyl chain composition. ACS Infect Dis 3(11):797-801. https://doi.org/10.1021/acsinfecdis.7b00138

Tello M, Kuzuyama T, Heide L, Noel JP, Richard SB (2008) The ABBA family of aromatic prenyltransferases: broadening natural product diversity. Cell Mol Life Sci 65(10):1459-1463. https://doi.org/10. 1007/s00018-008-7579-3

Vilhena C, Bettencourt A (2012) Daptomycin: a review of properties, clinical use, drug delivery and resistance. Mini-Rev Med Chem 12:202-209. https://doi.org/10.2174/1389557511209030202

Winkelblech J, Liebhold M, Gunera J, Xie X, Kolb P, Li S-M (2015) Tryptophan $C_{5-,}$ C6-, and $C 7$-prenylating enzymes displaying a preference for C- 6 of the indole ring in the presence of unnatural dimethylallyl diphosphate analogues. Adv Synth Cat 357(5):975986. https://doi.org/10.1002/adsc.201400958

Yin N, Li J, He Y, Herradura P, Pearson A, Mesleh MF, Mascio CT, Howland K, Steenbergen J, Thorne GM, Citron D, Van Praagh AD, Mortin LI, Keith D, Silverman J, Metcalf C (2015) Structure-activity relationship studies of a series of semisynthetic lipopeptides leading to the discovery of surotomycin, a novel cyclic lipopeptide being developed for the treatment of Clostridium difficile-associated diarrhea. J Med Chem 58(12):5137-5142. https://doi.org/10.1021/acs. jmedchem.5b00366

You J, Dai H, Chen Z, Liu G, He Z, Song F, Yang X, Fu H, Zhang L, Chen X (2010) Trichoderone, a novel cytotoxic cyclopentenone and cholesta-7, 22-diene-3 $\beta, 5 \alpha, 6 \beta$-triol, with new activities from the marine-derived fungus Trichoderma sp. J Ind Microbiol Biotechnol 37(3):245-252. https://doi.org/10.1007/s10295-009-0667-z

Yu H, Liebhold M, Xie X, Li SM (2015) Tyrosine $O$-prenyltransferases TyrPT and SirD displaying similar behavior toward unnatural alkyl or benzyl diphosphate as their natural prenyl donor dimethylallyl diphosphate. Appl Microbiol Biotechnol 99(17):7115-7124. https://doi.org/10.1007/s00253-015-6452-1

Zhang T, Muraih JK, Tishbi N, Herskowitz J, Victor RL, Silverman J, Uwumarenogie S, Taylor SD, Palmer M, Mintzer E (2014) Cardiolipin prevents membrane translocation and permeabilization by daptomycin. J Biol Chem 289(17):11584-11591. https://doi.org/ 10.1074/jbc.M114.554444

Publisher's note Springer Nature remains neutral with regard to jurisdictional claims in published maps and institutional affiliations. 